\title{
Impact and inhibitory mechanism of phenolic compounds on the formation of toxic Maillard reaction products in food
}

\author{
Jing TENG, Xiaoqian HU, Ningping TAO, Mingfu WANG (ه) \\ College of Food Science and Technology, Shanghai Ocean University, Shanghai 201306, China
}

\begin{abstract}
As one of the dominant reactions occurring during thermal treatment of food, the Maillard reaction not only leads to the formation of aroma, browning color and taste compounds, but also contributes to the formation of some unpleasant toxic substances including acrylamide, heterocyclic amines and advanced glycation end products. Polyphenols, one of the most abundant antioxidants in the human diet, are contained in different kinds of foods. In this review, some recent studies on the impact of dietary polyphenols on the formation of acrylamide, heterocyclic amines and advanced glycation end products formed during the Maillard reaction are summarized, including the research work conducted with both chemical model systems and real food model systems; the possible inhibitory mechanisms of different polyphenols are also summarized and discussed in this review. Basically we found that some dietary polyphenols not only scavenge free radicals, but also react with reactive carbonyl species, thus lowering the formation of toxic Maillard reaction products. This review provides a useful theoretical foundation for the application of polyphenols in food safety, and suggests some directions for further study of natural products as inhibitors against the formation of toxic substances in thermally processed food.
\end{abstract}

Keywords advanced glycation end products, acrylamide, food safety, heterocyclic amine, Maillard reaction, polyphenols

\section{Introduction}

Phenolic compounds are naturally occurring secondary metabolites in plants. They are a group of phytochemicals with at least one aromatic ring and one or more substituted hydroxyl groups ${ }^{[1]}$. Phenolics containing two or more hydroxyl groups on the aromatic rings are called as

Received July 28, 2017; accepted September 3, 2017

Correspondence: mfwang@shou.edu.cn polyphenols. Polyphenols are considered to be one of the most abundant antioxidants in human diets and are found in a wide range of food and beverages, such as fruits, vegetables, spices, herbs, grains, red wine, tea and coffee $^{[2,3]}$. They are health-promoting or diseasepreventing agents with many biological functions such as antioxidative, anti-inflammatory, neuro-protective, cardioprotective, cancer chemopreventive and anti-obesity activity ${ }^{[4,5]}$. These properties can largely be attributed to their free radical scavenging/direct antioxidative capability ${ }^{[1]}$; polyphenols can also help to activate the endogenous defense systems and modulate different cellular signaling pathways.

Currently, over 8000 phenolic compounds have been identified in different plants. Based on the number of aromatic rings as well as structural elements connecting rings to one another ${ }^{[6]}$, they can be classified into a few groups including simple phenols, flavonoids, lignans and stilbenes $^{[7]}$. Among them, simple phenols can be divided into several subgroups including derivatives of cinnamic acid and benzoic acid ${ }^{[8]}$. About one third of the total phenolic compound intake is simple phenols while flavonoids also account for a major part of the intake of total phenolics ${ }^{[1]}$. Flavonoids, with more than 4000 varieties identified, comprise the most studied group of polyphenols. The basic skeleton for flavonoids is based upon a 15-carbon skeleton consisting of two aromatic rings bound together by a heterocyclic pyran ring ${ }^{[9]}$. Depending on the level of oxidation, saturation and pattern of substitution of the $\mathrm{C}$ ring, flavonoids can be divided into a variety of subclasses listed as anthocyanins, flavanols, flavanones, flavones, flavonols and isoflavones ${ }^{[8]}$. Figure 1 shows the structures of some common dietary polyphenols.

The Maillard reaction is the chemical reaction between reducing sugars and amino compounds. It was first described by Louis-Camille Maillard in $1912^{[10]}$, and in 1953, Hodge proposed the first comprehensive scheme for the Maillard reaction ${ }^{[11]}$. Currently it is widely accepted that the Maillard reaction, one of the dominant reactions occurring during thermal processing of food, leads to the 
<smiles>O=C(O)/C=C/c1ccc(O)c(O)c1</smiles><smiles>COc1cc(CC(CO)C(CO)Cc2ccc(O)c(OC)c2)ccc1O</smiles>

Lignans<smiles>O=C(O[C@H]1Cc2c(O)cc(O)cc2O[C@H]1c1cc(O)c(O)c(O)c1)c1cc(O)c(O)c(O)c1</smiles>

Epigallocatechin gallate (EGCG)<smiles>Oc1cc(O)c2c(c1)OC(c1ccc(O)c(O)c1)C(O)C2c1c(O)cc(O)c2c1OC(c1ccc(O)c(O)c1)C(c1c(O)cc(O)c3c1OC(c1ccc(O)c(O)c1)C(O)C3)C2O</smiles>

Procyanidin

Fig. 1 Structures of some popular dietary polyphenols

formation of aroma, color and taste compounds ${ }^{[12]}$. In addition, recent studies have shown that certain categories of Maillard reaction products (MRPs) have a nonnegligible hazardous impact on human health, including acrylamide ${ }^{[13]}$ produced in starch-rich processed foods, heterocyclic amines occurred in protein-rich cooked foods ${ }^{[14]}$ and advanced glycation end products (AGEs) in baking and dairy products ${ }^{[15,16]}$. They are known to be mutagens and carcinogens, therefore, strategies including changing food processing conditions and reaction precursors, and the addition of food additives are being adopted to lower or inhibit the formation of these toxic MPRs.

The natural ingredients extracted from plants, especially polyphenols, have been widely used for a long time as dietary or nutritional supplements. As they not only have low toxicity and side effects, but also have their own biological effects and are increasingly studied for their potential inhibitory effects on the formation of harmful MRPs $^{[17-19]}$. Here, we review for the first time the effects of polyphenols on the formation of toxic MRPs. Studies using chemical and real food model systems to evaluate the inhibitory effect of selected phenolics and phenolic extracts on the formation of acrylamide, heterocyclic amines and AGEs, as well as the possible inhibitory mechanisms, are summarized. They should help provide a theoretical foundation for the potential application of polyphenols in food safety, and lead to the further study of inhibitory effects of natural products and mechanisms of MRP toxicity.

\section{Impacts of polyphenols on the formation of acrylamide in the Maillard reaction}

In 2002, considerable levels of acrylamide as a contaminant in heat-processed, carbohydrate-rich foods were found by Swedish scientists ${ }^{[20,21]}$. In a mechanistic view, acrylamide was found to be formed from the reaction of reducing sugars or reactive dicarbonyls with an amino acid, asparagine via the Maillard reaction ${ }^{[22,23]}$, which is known as the asparagine route ${ }^{[24]}$. 3-Aminopropionamide (3-APA) (Fig. 2), an important intermediate proposed in the reaction pathway, was found to lead to a high yield of acrylamide: $60 \%$ (molar ratio) after deamination ${ }^{[24,25]}$.

Acrylamide is a concern in human diets as it can cause cancer in laboratory animals at high doses, and has been classified by the International Agency for Research on Cancer (IARC) as a possible human carcinogen ${ }^{[26,27]}$. Reports on its presence in some foods widely consumed daily, especially starch-rich food products like crispy breads and fried potatoes, have raised global concerns due to the fact that humans might be exposed to significant quantities of acrylamide in the long run ${ }^{[20,27,28]}$. Studies have been conducted on the effects of polyphenols, including phenolic extracts from plants or pure 


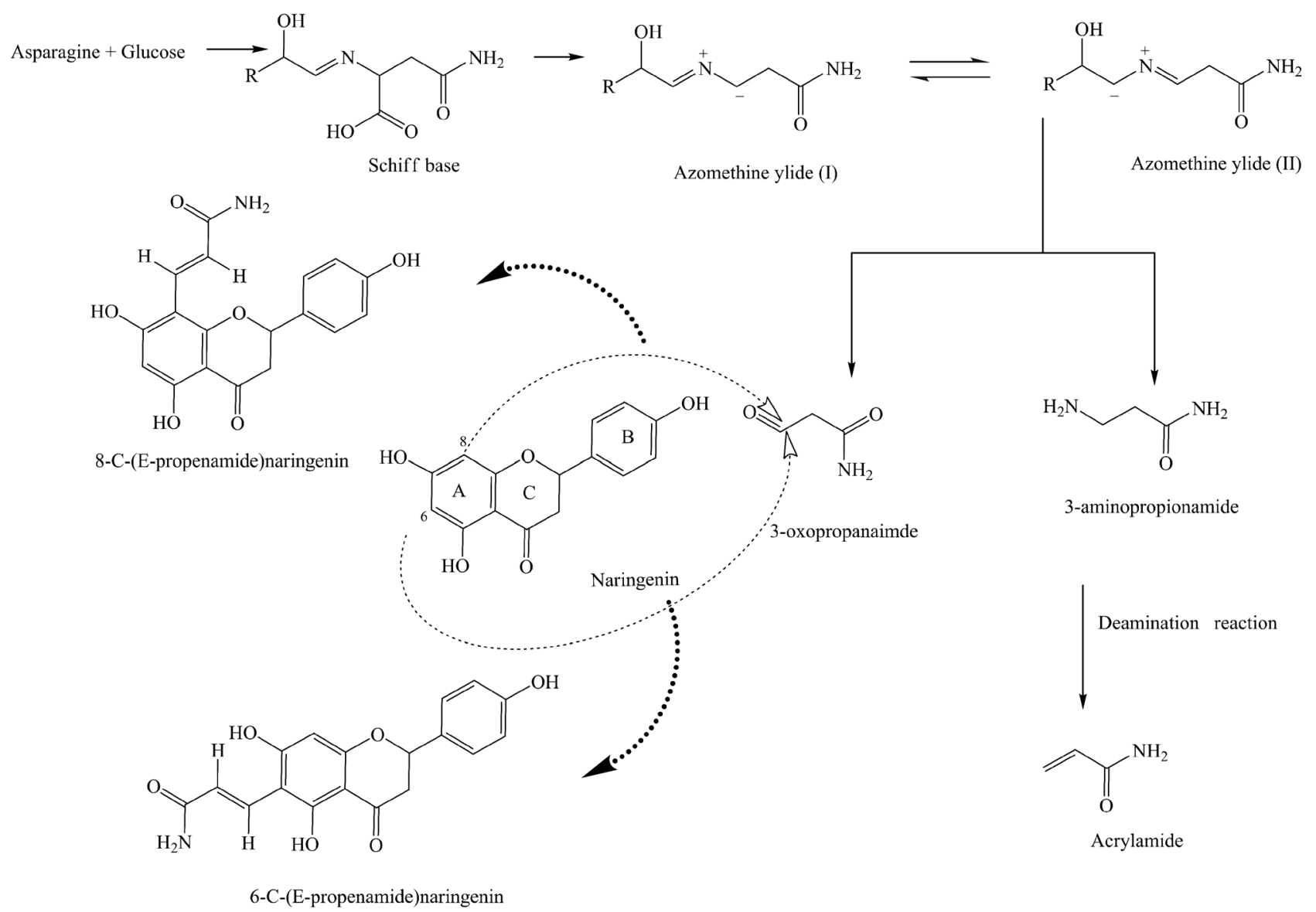

Fig. 2 The postulated pathway for acrylamide formation via the Maillard reaction and probable inhibitory mechanism of polyphenols against the formation of acrylamide

polyphenols, on acrylamide formation during thermal processing.

Researchers have used both chemical model systems and real food models to conduct studies ${ }^{[21,24,26,27,29,30]}$. When it comes to chemical models, the asparagine-glucose system has been the most studied. For instance, Cheng et al. ${ }^{[27]}$ used the asparagine-glucose chemical reaction model $\left(25 \mathrm{mmol} \cdot \mathrm{L}^{-1}\right.$ in $10 \mathrm{~mL}$ of $\mathrm{pH} 7.0$ phosphate buffer $)$ to study the effect of naringenin $\left(5,10\right.$ and $\left.25 \mathrm{mmol} \cdot \mathrm{L}^{-1}\right)$ on the formation of acrylamide under $128^{\circ} \mathrm{C}$ for $60 \mathrm{~min}$, and the results indicated that naringenin significantly and nonlinearly (i.e., dose-dependent) inhibited formation of acrylamide. Researchers also used potato-based equimolar asparagine-glucose/reducing sugar model system which were designed as described below ${ }^{[24,29]}$. Commonly, at the beginning, the concentrations of glucose/(glucose and fructose) and asparagine in the potato matrix were measured by HPLC, after which the additions of glucose (glucose and fructose) and asparagine were calculated, to produce an equimolar asparagine-glucose/reducing sugar Maillard reaction model system. Cheng found that both catechins and esterified catechins inhibit the formation of acrylamide effectively in this model; and with additions reaching $10^{-9} \mathrm{~mol} \cdot \mathrm{L}^{-1}$ (in $0.1 \mathrm{~mol} \cdot \mathrm{L}^{-1} \mathrm{pH} 6.8$ phosphate buffer), the maximum reduction rate was obtained ${ }^{[21]}$.

Using a real food model system, Xu et al. ${ }^{[26]}$ studied the effect of muscadine grape polyphenol extract on the reduction of acrylamide in a potato chip model, which was prepared by immersing potato slices in $0.025 \%, 0.05 \%$ and $0.1 \%$ solution polyphenol extract of Carlos grape skin for $60 \mathrm{~min}$ before frying $\left(175^{\circ} \mathrm{C}, 5 \mathrm{~min}\right)$. This resulted in a $60.3 \%$ reduction acrylamide concentration when the immersion solution contained $0.1 \%$ of the extract. Also, no significant relationship was found between antioxidant activity of polyphenols and inhibitory effect against the formation of acrylamide ${ }^{[26]}$. In another study, a biscuit model was built by Oral et al. ${ }^{[30]}$, in which they added different kinds of phenolics, including caffeic acid, chlorogenic acid, ellagic acid, epicatechin, oleuropein, punicalagin and tyrosol $\left(30 \mu \mathrm{mol} \cdot \mathrm{L}^{-1}\right.$ per $100 \mathrm{~g}$ flour, respectively) to dough that was baked for $15 \mathrm{~min}$ at $185^{\circ} \mathrm{C}$. All the tested phenolic compounds reduced acrylamide formation by $10.3 \%$ to $19.2 \%$.

Some researchers have studied the structure/activity relationship of different polyphenols against acrylamide formation. For example, flavone C-glycosides were found 
to be more efficient at reducing acrylamide formation than the O-glycosides with the same aglycone ${ }^{[29]}$, and for flavonoid structures, 5,7(meta)-dihydroxyls in A cycle, $3^{\prime}, 4^{\prime}$ (ortho)-dihydroxyls in B cycle and 3-hydroxyl or hydroxyls of 3-gallate in $\mathrm{C}$ cycle, were found to be effective in acrylamide reduction ${ }^{[24]}$. However, the inhibitory mechanism of polyphenols against acrylamide has been rarely reported. One of the possible mechanisms is that some polyphenols might react with precursors of acrylamide directly, in other words, they might divert those significant precursors from the acrylamide formation pathway, thus lowering acrylamide production. For example, Cheng et al. ${ }^{[27]}$ found that naringenin reacts with 3-oxopropanamide (Fig. 2), a Maillard intermediate, giving rise to new derivatives including 8-C-(E-propenamide)naringenin and 6-C-(E-propenamide)naringenin. The reaction between naringenin and 3-oxopropanamide actually caused depletion of this amide source, which might further promote the flux of azomethine ylide toward 3-oxopropanamide. Therefore, the conversion of azomethine ylide in the 3-APA pathway might be inhibited to some extent, so the formation of acrylamide might be suppressed $^{[27]}$.

\section{Impacts of polyphenols on the formation of heterocyclic amines in the Maillard reaction}

In the late 1970s, Japanese scientists found mutagenic activity on the charred surface of broiled beef and fish ${ }^{[31]}$, and these food-derived mutagens were isolated and characterized as heterocyclic aromatic amines (HAs) $)^{[14]}$. HAs are polycyclic aromatic molecules, and can be formed during the thermal processing of protein-rich foods, especially meat and fish, via the Maillard reaction with amino acids, sugars and creatinine as precursors ${ }^{[32,33]}$. Currently, over 25 different kinds of HAs have been identified in various types of cooked food ${ }^{[34]}$. Among them, 2-amino-1-methyl-6-phenylimidazo[4,5-b]pyridine (PhIP) (Fig. 3a), was found to be the most abundantly formed under ordinary household cooking procedures ${ }^{[35]}$. Also, 2-amino-3,8-dimethylimidazo[4,5-f]quinoxaline (MeIQx) and 2-amino-3,4,8-dimethylimidazo[4,5-f]quinoxaline (4,8-DiMeIQx) (Fig. 3a) have been reported as abundant HAs formed in foods like bacon, beef, fish and poultry ${ }^{[33]}$.

When considering HA formation pathway, using PhIP as (a)<smiles>Cc1cnc2ccc3c(nc(N)n3C)c2n1</smiles><smiles>Cc1cnc2cc(C)c3c(nc(N)n3C)c2n1</smiles>

2-Amino-3,4,8-dimethylimidazo[4,5-f]quinoxaline<smiles>Cn1c(N)nc2ncc(-c3ccccc3)cc21</smiles>

(b)

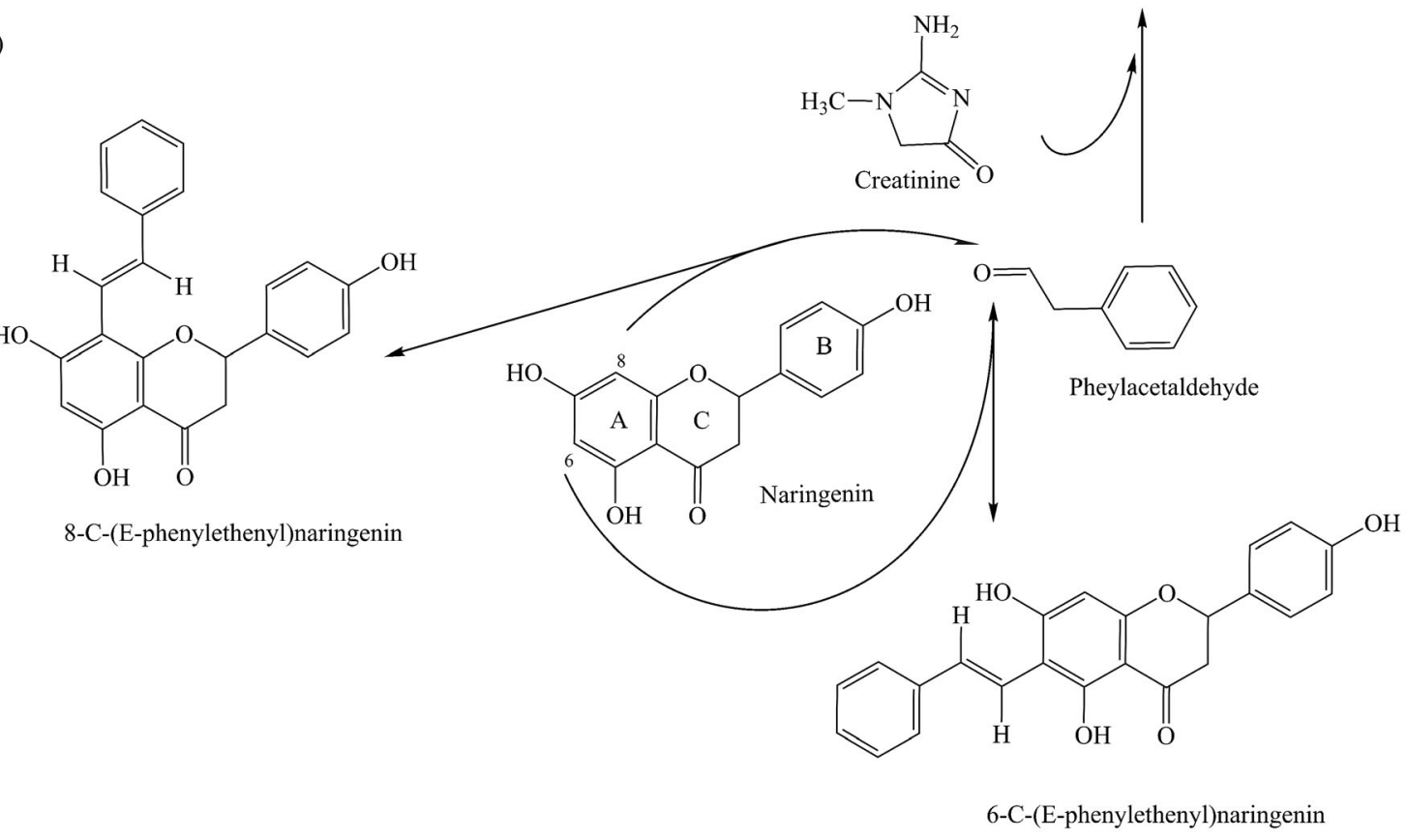

Fig. 3 (a) Structures of some abundant heterocyclic amines formed during thermal processing of food; (b) the probable inhibitory mechanism of selected polyphenolics against the formation of 2-amino-1-methyl-6-phenylimidazo[4,5-b]pyridine. 
an example, phenylalanine, carbohydrates and creatinine have been identified to be the main precursors of $\mathrm{PhIP}$, which was considered as a coproduct in the Maillard reaction ${ }^{[36]}$. Several steps were involved in this reaction, while the formation of phenylacetaldehyde from phenylalanine degradation (Streker degradation) and its subsequent reaction with creatinine might be the key steps ${ }^{[37]}$. Some HAs have been found to be highly carcinogenic and mutagenic $^{[38]}$, and as an example, the IARC concluded that there was sufficient evidence from studies of laboratory animals for PhIP to be consider to be carcinogenic ${ }^{[36]}$.

With the knowledge of the high mutagenicity and carcinogenicity of HAs, increasing efforts were made by researchers to test whether polyphenols could show inhibitory effects on HA formation in both chemical reaction model and real food models systems ${ }^{[33,39-44]}$. In Cheng's chemical model system study ${ }^{[39]}, 12$ varieties of food-sourced antioxidative phenolic compounds were examined for their relationship between efficacy against $\mathrm{PhIP}$ formation and radical scavenging capacity. The result showed that the correlation between radical scavenging activity (assessed by TEAC assay) and the inhibitory effect against PhIP formation in phenylalanine-glucose-creatinine chemical model system was quite poor. In addition, bioactivity-guided separation and purification using PhIPand a MeIQx-producing model were applied to determine the components in apple extract responsible for the inhibition of HA formation. It was found that apple proanthocyanidins inhibited both PhIP and MeIQx formation, but only phloridzin showed inhibitory activity against PhIP formation, while chlorogenic acid significantly enhanced the formation of PhIP, though it could inhibit MeIQx formation ${ }^{[40]}$. In Zhang's study ${ }^{[42]}$, the inhibitory effects of flavonoids (apigenin, fisetin, hesperetin, homoorientin, isorhamnetin, isovitexin, luteolin, orientin, vitexin) and a bamboo leaf extract (AOB) on PhIP production were tested in a phenylalanine-creatinine model systems $\left(120^{\circ} \mathrm{C}, 2 \mathrm{~h}\right)$, and the results indicated that the tested flavonoids and AOB had significant dosedependent inhibitory effects against PhIP formation.

For researches using real food model systems, beef patty has been the most popular food model for evaluating potential inhibitors of HA formation. The inhibitory effects of different kinds of dietary flavonoids, including apigenin, epigallocatechin gallate, genistein, kaempferol, luteolin, phlorizin and quercetin on HA formation were studied by Zhu et al. ${ }^{[4]}$, and results showed that most of these flavonoids gave significant reduction of both PhIP and total HAs. Epigallocatechin gallate and quercetin were the most effective against the formation of both PhIP (60\%$80 \%)$ and total HAs $(55 \%-70 \%)$. In a study by Sabally et al. ${ }^{[33]}$, the capability of polyphenol-rich dried apple peel extract (DAPP) against the formation of HAs in beef patty model was assessed. DAPP was applied on the surface or mixed inside the patties, then MeIQx, PhIP and 4,8-DiMeIQx were quantified after frying. DAPP at
$0.3 \%$ on the surface inhibited $(P<0.05)$ HA formation by $83 \%$ for PhIP, $68 \%$ for MeIQx and $56 \%$ for 4,8 -DiMeIQx, compared to $60 \%, 41 \%$ and $21 \%$, respectively, for the group in which DAPP was mixed inside the patties $^{[33]}$.

Some efforts have also made to study the mechanism of the inhibition of HA formation by phenolic compounds, with two mechanism currently suggested. Using PhIP as an example, on one hand, the inhibitory effects might be attributed to their abilities to scavenge or trap phenylacetaldehyde, a chief intermediate in the pathway to the formation of PhIP, via polyphenol-phenylacetaldehyde adduct formation ${ }^{[41,44]}$. Cheng et al. ${ }^{[45]}$ found that reduction in phenylacetaldehyde concentration by naringenin was dose-dependent, which might be due to the interaction between phenylacetaldehyde with naringenin to form adducts, nevertheless the availability of phenylacetaldehyde for PhIP formation was reduced. The two adducts found in their research were 8-C-(E-phenylethenyl)naringenin and 6-C-(E-phenylethenyl)naringenin (Fig. 3b $)^{[45]}$, with the latter known to suppress colorectal cancer growth through cyclooxygenase-1 inhibition ${ }^{[46]}$. Norartocarpetin is another kind of polyphenol that can suppress PhIP formation in roast beef patties and form norartocarpetinphenylacetaldehyde adducts. The adduct 8-C-(E-phenylethenyl)norartocarpetin was discovered by Zheng et al. ${ }^{[43]}$ to have anticancer potential via intrinsic caspase-dependent and cell context-dependent MAPKs pathways. The postulated pathways for (-)-epigallocatechin-3-gallate (EGCG) inhibition of PhIP formation were also considered to be through EGCG-phenylacetaldehyde adduct formation $^{[41]}$. In addition, adducts such as 8/6-C-(E-phenylethenyl)qucercetin, 8/6-C-(E-phenylethenyl)apigenin, 8/6-C(E-phenylethenyl)kaempferol, 8/6-C-(E-phenylethenyl) luteolin, were isolated from the chemical reaction system by preparative HPLC and identified by LC-MS in Zhu's work $^{[44]}$. These studies suggest that a lot of different phenolics/flavonoids can directly trap phenylacetaldehyde, thus lower the formation of mutagenic PhIP. Another possible mechanism proposed by some researchers was that the inhibitory effects of polyphenols/flavonoids on HA formation were probably achieved by scavenging of free radicals produced during thermal treatment, or scavenging of both free radical and reactive carbonyl species such as phenylacetaldehyde simultaneously, which might have a synergistic effect on HA inhibition ${ }^{[42]}$.

\section{Impacts of polyphenols on the formation of advanced glycation end products in the Maillard reaction}

The initial understanding of AGEs was from the observation of glycosylated hemoglobin in diabetics. Currently it is well accepted that AGEs can be formed through different pathways such as glucose autoxidation, lipid peroxidation, 
glycolysis and the polyol pathway ${ }^{[47]}$ in the human body. In addition, the Maillard reaction occurring during food processing was also demonstrated to serve as an exogenous factor for the formation of AGEs. In other words, AGEs can be produced when amino acids, peptides or protein reacted with reducing sugars ${ }^{[48]}$. After Schiff base formation and Amadori rearrangement, AGEs can be formed through either oxidative reaction or non-oxidative reaction with these two types of intermediate products. On the other hand, Amadori products can dehydrate and rearrange to form reactive dicarbonyl compounds (Fig. 4) ${ }^{[49]}$, such as methylglyoxal (MGO) and glyoxal (GO), which are typical $\alpha$-dicarbonyl compounds, and can actually also serve as significant precursors for the formation of AGEs, through their subsequent reactions with protein amino, sulfhydryl and guanidine functional groups $^{[50-52]}$. According to their fluorescence and crosslinking properties, AGEs can be divided into four categories, which are fluorescent crosslinking compounds, non-fluorescent crosslinking compounds, fluorescent noncrosslinking compounds and non-fluorescent non-crosslinking compounds.

$\mathrm{N}^{\mathrm{e}}$-(carboxymethyl)lysine (CML) (Fig. 4), an extensively studied and well-characterized AGE formed in food processing or in vivo, can be formed by both the reaction of -amino group in lysine with GO and the oxidation of fructoselysine ${ }^{[53]}$, while $\mathrm{N}^{\mathrm{e}}$-(carboxyethyl) lysine can be produced primarily by the reaction of MGO and lysine residues ${ }^{[54]}$. Recently, the accumulation of AGEs in vivo has been considered as a major pathogenic process in diabetic complications, including retinopathy, cataracts and other health disorders, such as the growth and metastasis of malignant tumors, Alzheimer's disease, inflammation and atherosclerosis, as well as in normal aging ${ }^{[55,56]}$. Therefore, in recent years, in order to seek ways to reduce AGE formation during thermal processing of food, investigations have been conducted with various plant extracts to observe their impact on AGEs formation and some suppression activity has been attributed to the large number of phenolic compounds they contain ${ }^{[53]}$.

A range of polyphenols have been reported for their inhibitory effects against AGE formation in both chemical models and food models ${ }^{[19,42,51,53,57,58]}$. In studies conducted using chemical models, Sang et al. ${ }^{[57]}$ found that below $37^{\circ} \mathrm{C}$ at $\mathrm{pH} 7.4$, EGCG can trap GO and MGO, two intermediates for AGE formation, to form mono- and diGO or MGO adducts, and the data from LC/MS and NMR indicated that $\mathrm{C} 6$ and $\mathrm{C} 8$ on the A-ring of EGCG were the key positions for trapping (Fig. 4). In Liu's research ${ }^{[51]}$, quercetin could effectively inhibit the formation of both GO and MGO in a time-dependent manner in a glucose/ lysine Maillard reaction model system, and the di-MGO

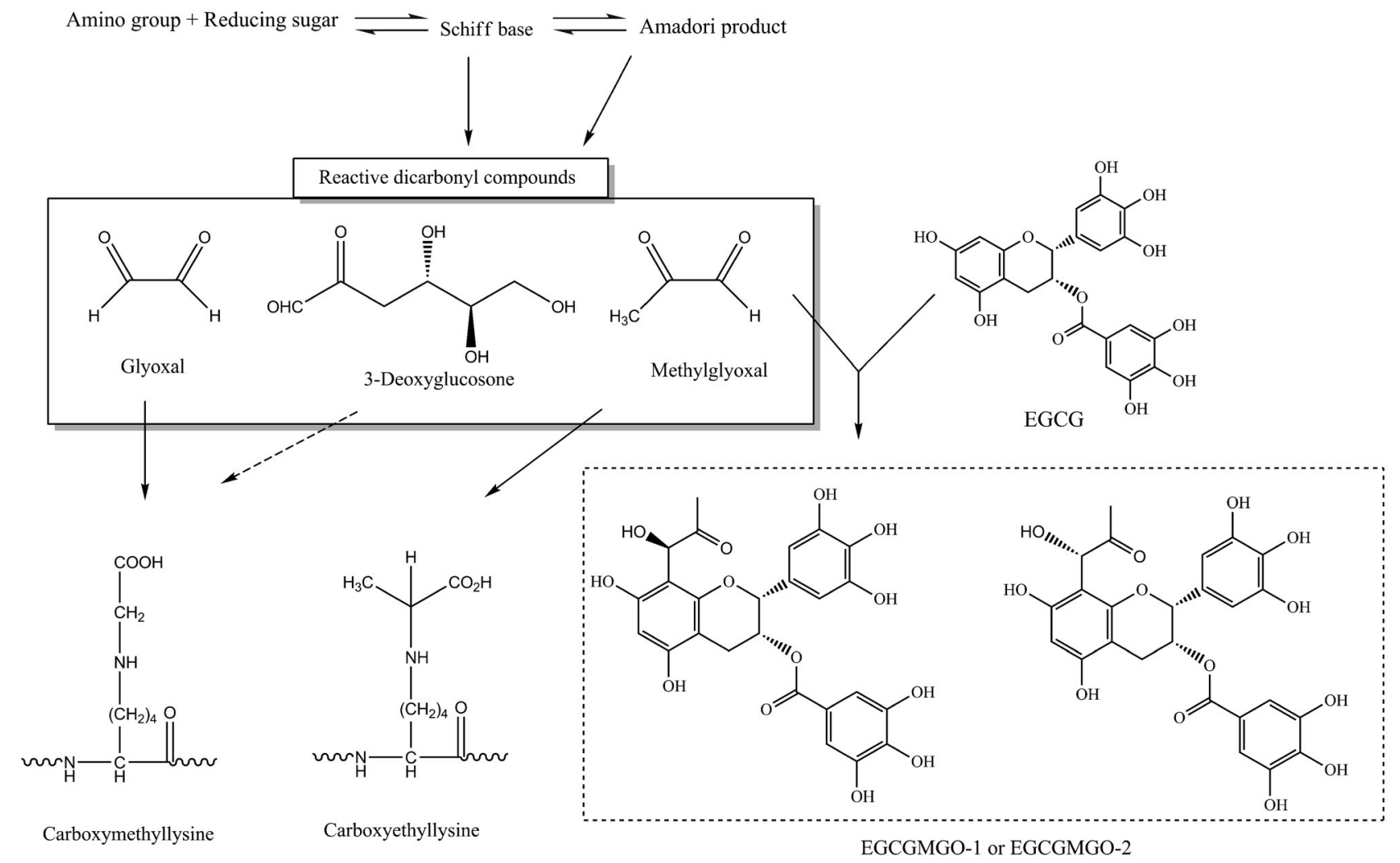

Fig. 4 The possible pathways for AGE formation as well as inhibitory strategy and structures of some typical reactive carbonyl compounds and AGEs 
quercetin adduct could further trap MGO to produce tri-MGO adducts. To sum up, MGO quercetin adducts retained the trapping ability of reactive dicarbonyl species as well as antioxidant activity.

Effects of polyphenols on AGE formation in food models have also been reported. Peng et al. ${ }^{[58]}$ discovered that grape seed extract (95\% proanthocyanidins including epicatechin and catechin) can reduce CML in bread in a dose-dependent manner and the addition of 1.2 and $2 \mathrm{~g} \cdot \mathrm{kg}^{-1}$ to bread led to more than $30 \%$ and $50 \%$ reduction of CML content of bread crust, respectively. Zhang et al. ${ }^{[42]}$ studied the impact of chlorogenic acid, epicatechin, naringenin, quercetin and rosmarinic acid on AGE formation in a biscuit model $(0.25 \% \mathrm{w} / \mathrm{w}$ of individual phenolic compound in $7.5 \mathrm{~g}$ of white flour), and the results showed that the polyphenols tested, especially quercetin, were inhibitory to the formation of both reactive carbonyl species and total fluorescent AGEs. In addition, phenolic compounds including caffeic acid, catechin, ferulic acid, gallic acid and quercetin were studied for their effects on AGEs formation by Mildner-Szkudlarz et al. ${ }^{[19]}$, with a bread model system and four levels of addition $(1,5,10$ and $20 \mathrm{~g} \cdot \mathrm{kg}^{-1}$ of flour). These phenolic compounds, except ferulic acid, were found to significantly reduce CML (31.8\% to $87.6 \%$ ) even at the lowest concentration, while the strongest inhibitory effect on FA (62\%) appeared only if the addition was at least $10 \mathrm{~g} \cdot \mathrm{kg}^{-1}$ of flour.

As for the potential inhibitory mechanisms of phenolic compounds on AGE formation during thermal processing of food, two mechanisms have currently been suggested. First, free radicals formed during glycation might be scavenged by polyphenols due to their antioxidant activities, therefore subsequent AGE formation is inhibited. Second, the inhibition might also occur through the trapping of reactive carbonyl species by phenolic compounds ${ }^{[53]}$, or there might be a combined effect of both for the inhibitory actions. Several proanthocyanidins from cinnamon bark have been identified as inhibitors of specific AGE representatives, which were largely attributed to both their carbonyl scavenging abilities as well as antioxidant activities ${ }^{[59]}$. Another report also indicated that quercetin has a dual role inhibiting MGO formation by scavenging free radicals produced in the system, while concurrently trapping MGO to form adducts $^{[51]}$.

\section{Conclusions}

The Maillard reaction leads to the formation of not only aroma, browning color and taste compounds of thermally processed foods, but also some unpleasant toxic substances including acrylamide, heterocyclic amines and advanced glycation end products. Evidence from chemical reaction model systems and real food models has shown that some polyphenols can serve as potent inhibitors to reduce toxic
MRP formation during thermal processing of food. The inhibitory mechanisms of polyphenols against toxic MRP formation have usually been attributed to free radical scavenging activities, with recent research indicating some additional mechanisms. For acrylamide suppression, some polyphenols can react with 3-oxopropanamide directly, to divert it from pathways with a 3-APA deamination reaction, resulting in less acrylamide formation. For HAs, using PhIP as an example, research has shown that the inhibitory effects can be attributed to trapping phenylacetaldehyde via the formation of polyphenolphenylacetaldehyde adduct. Hopefully, the adducts formed via polyphenol trapping by phenylacetaldehyde might have extra health benefits such as anticancer capabilities. When it comes to AGE inhibition, both polyphenol antioxidant activities and carbonyl scavenging capacities contribute to the reduction of AGEs, so polyphenols may not only alleviate AGE-caused health disorders, but also prevent detrimental RCS-induced symptoms. To sum up, in thermal processing of food, some polyphenols have significant potential to lower the formation of toxic MRPs and may also participate in the Maillard reaction to form beneficial substances.

Acknowledgements This work was partially supported by National Natural Science Foundation of China (31671821)

Compliance with ethics guidelines Jing Teng, Xiaoqian Hu, Ningping Tao, and Mingfu Wang declare that they have no conflicts of interest or financial conflicts to disclose.

This article is a review and does not contain any studies with human or animal subjects performed by any of the authors.

\section{References}

1. Han X, Shen T, Lou H. Dietary polyphenols and their biological significance. International Journal of Molecular Sciences, 2007, 8 (9): 950-988

2. Scalbert A, Manach C, Morand C, Rémésy C, Jiménez L. Dietary polyphenols and the prevention of diseases. Critical Reviews in Food Science and Nutrition, 2005, 45(4): 287-306

3. Hua Z, Rong T. Dietary polyphenols, oxidative stress and antioxidant and anti-inflammatory effects. Current Opinion in Food Science, 2016, 8: 33-42

4. Landete J M. Updated knowledge about polyphenols: functions, bioavailability, metabolism, and health. Critical Reviews in Food Science and Nutrition, 2012, 52(10): 936-948

5. Wang S, Moustaid-Moussa N, Chen L, Mo H, Shastri A, Su R, Bapat P, Kwun I, Shen C L. Novel insights of dietary polyphenols and obesity. Journal of Nutritional Biochemistry, 2014, 25(1): $1-18$

6. Butterfield D, Castegna A, Pocernich C, Drake J, Scapagnini G, Calabrese V. Nutritional approaches to combat oxidative stress in Alzheimer's disease. Journal of Nutritional Biochemistry, 2002, 13 (8): 444-461 
7. Spencer J P, Abd El Mohsen M M, Minihane A M, Mathers J C. Biomarkers of the intake of dietary polyphenols: strengths, limitations and application in nutrition research. British Journal of Nutrition, 2008, 99(1): 12-22

8. Pandey K B, Rizvi S I. Plant polyphenols as dietary antioxidants in human health and disease. Oxidative Medicine and Cellular Longevity, 2009, 2(5): 270-278

9. Kumar S, Pandey A K. Chemistry and biological activities of flavonoids: an overview. Scientific World Journal, 2013, 2013(1112): 162750

10. Hellwig M, Henle T. Baking, ageing, diabetes: a short history of the Maillard reaction. Angewandte Chemie International Edition, 2014, 53(39): 10316-10329

11. Hodge J E. Dehydrated foods. Chemistry of browning reactions in model systems. Journal of Agricultural and Food Chemistry, 1953, 1(1): $625-651$

12. Martins S I F S, Jongen W M F, Boekel M A J S V. A review of Maillard reaction in food and implications to kinetic modelling. Trends in Food Science \& Technology, 2001, 11(9-10): 364-373

13. Mottram D S, Wedzicha B L, Dodson A T. Acrylamide is formed in the Maillard reaction. Nature, 2002, 419(6906): 448-449

14. Cheng K W, Chen F, Wang M. Heterocyclic amines: chemistry and health. Molecular Nutrition \& Food Research, 2006, 50(12): 11501170

15. Ahmed N, Mirshekar-Syahkal B, Kennish L, Karachalias N, BabaeiJadidi R, Thornalley P J. Assay of advanced glycation endproducts in selected beverages and food by liquid chromatography with tandem mass spectrometric detection. Molecular Nutrition \& Food Research, 2005, 49(7): 691-699

16. Goldberg T, Cai W, Peppa M, Dardaine V, Baliga B S, Uribarri J, Vlassara H. Advanced glycoxidation end products in commonly consumed foods. Journal of the American Dietetic Association, 2004, 104(8): 1287-1291

17. Zhang Y, Xu W, Wu X, Zhang X, Zhang Y. Addition of antioxidant from bamboo leaves as an effective way to reduce the formation of acrylamide in fried chicken wings. Food Additives and Contaminants, 2007, 24(3): 242-251

18. Zhang Y, Chen J, Zhang X, Wu X, Zhang Y. Addition of antioxidant of bamboo leaves (AOB) effectively reduces acrylamide formation in potato crisps and French fries. Journal of Agricultural and Food Chemistry, 2007, 55(2): 523-528

19. Mildner-Szkudlarz S, Siger A, Szwengiel A, Przygoński K, Wojtowicz E, Zawirska-Wojtasiak R. Phenolic compounds reduce formation of $\mathrm{N}(\varepsilon)$-(carboxymethyl)lysine and pyrazines formed by Maillard reactions in a model bread system. Food Chemistry, 2017, 231: $175-184$

20. Tareke E, Rydberg P, Karlsson P, Eriksson S, Törnqvist M. Analysis of acrylamide, a carcinogen formed in heated foodstuffs. Journal of Agricultural and Food Chemistry, 2002, 50(17): 4998-5006

21. Cheng J, Chen X, Lu H, Chen Q, Zhang Y. Antioxidant-related and kinetic studies on the reduction effect of catechins and esterified catechins on acrylamide formation in a microwave heating model system. RSC Advances, 2014, 4(82): 43378-43386

22. Lineback D R, Coughlin J R, Stadler R H. Acrylamide in foods: a review of the science and future considerations. Annual Review of Food Science and Technology, 2012, 3(1): 15-35
23. Haase N U, Grothe K H, Matthäus B, Vosmann K, Lindhauer M G. Acrylamide formation and antioxidant level in biscuits related to recipe and baking. Food Additives \& Contaminants: Part A, 2012, 29(8): 1230-1238

24. Zhang Y, Huang M, Wang Q, Cheng J. Structure-guided unravelling: phenolic hydroxyls contribute to reduction of acrylamide using multiplex quantitative structure-activity relationship modelling. Food Chemistry, 2016, 199: 492-501

25. Granvogl M, Schieberle P. Thermally generated 3-aminopropionamide as a transient intermediate in the formation of acrylamide. Journal of Agricultural and Food Chemistry, 2006, 54(16): 59335938

26. Xu C, Yagiz Y, Marshall S, Li Z, Simonne A, Lu J, Marshall M R. Application of muscadine grape (Vitis rotundifolia Michx.) pomace extract to reduce carcinogenic acrylamide. Food Chemistry, 2015, 182: 200-208

27. Cheng K W, Zeng X, Tang Y S, Wu J J, Liu Z, Sze K H, Chu I K, Chen $F$, Wang M. Inhibitory mechanism of naringenin against carcinogenic acrylamide formation and nonenzymatic browning in Maillard model reactions. Chemical Research in Toxicology, 2009, 22(8): 1483-1489

28. Friedman M. Chemistry, biochemistry, and safety of acrylamide. A review. Journal of Agricultural and Food Chemistry, 2003, 51(16): 4504-4526

29. Zhang Y, Chen X, Cheng J, Jin C, Zhang Y. The reduction effect of dietary flavone $\mathrm{C}$-and $\mathrm{O}$-glycosides on the formation of acrylamide and its correlation and prediction with the antioxidant activity of Maillard reaction products. RSC Advances, 2014, 4(46): 2414724155

30. Oral R A, Dogan M, Sarioglu K. Effects of certain polyphenols and extracts on furans and acrylamide formation in model system, and total furans during storage. Food Chemistry, 2014, 142: 423-429

31. Sugimura T, Nagao M, Kawachi T, Honda M, Yahagi T, Seino Y, Sato S, Matsukara N, Shirai A, Sawmura M, Matsumoto H. Mutagen-carcinogens in food, with special reference to highly mutagenic pyrolytic products in broiled foods. Origins of Human Cancer, 1977, 4: 1561-1577

32. Kizil M, Oz F, Besler $\mathrm{H}$ T. A review on the formation of carcinogenic/mutagenic heterocyclic aromatic amines. Food Processing \& Technology, 2011, 2(120): 2

33. Sabally K, Sleno L, Jauffrit J A, Iskandar M M, Kubow S. Inhibitory effects of apple peel polyphenol extract on the formation of heterocyclic amines in pan fried beef patties. Meat Science, 2016, 117: $57-62$

34. Alaejos M S, Afonso A M. Factors that affect the content of heterocyclic aromatic amines in foods. Comprehensive Reviews in Food Science and Food Safety, 2011, 10(2): 52-108

35. Zhang Y, Luo Z, Shao Z, Yu C, Wang S. Effects of antioxidants of bamboo leaves and flavonoids on 2-amino-1-methyl-6-phenylimidazo[4,5-b]pyridine (PhIP) formation in chemical model systems. Journal of Agricultural and Food Chemistry, 2014, 62(20): 47984802

36. Salazar R, Arámbula-Villa G, Hidalgo F J, Zamora R. Structural characteristics that determine the inhibitory role of phenolic compounds on 2-amino-1-methyl-6-phenylimidazo[4,5-b]pyridine (PhIP) formation. Food Chemistry, 2014, 151: 480-486 
37. Murkovic M, Weber H J, Geiszler S, Fröhlich K, Pfannhauser W. Formation of the food associated carcinogen 2-amino-1-methyl-6phenylimidazo[4,5-b]pyridine (PhIP) in model systems. Food Chemistry, 1999, 65(2): 233-237

38. Zeng M, He Z, Zheng Z, Qin F, Tao G, Zhang S, Gao Y, Chen J. Effect of six Chinese spices on heterocyclic amine profiles in roast beef patties by ultra performance liquid chromatography-tandem mass spectrometry and principal component analysis. Journal of Agricultural and Food Chemistry, 2014, 62(40): 9908-9915

39. Cheng K W, Chen F, Wang M. Inhibitory activities of dietary phenolic compounds on heterocyclic amine formation in both chemical model system and beef patties. Molecular Nutrition \& Food Research, 2007, 51(8): 969-976

40. Cheng K W, Wu Q, Zheng Z P, Peng X, Simon J E, Chen F, Wang $M$. Inhibitory effect of fruit extracts on the formation of heterocyclic amines. Journal of Agricultural and Food Chemistry, 2007, 55(25): 10359-10365

41. Cheng K W, Wong C C, Chao J, Lo C, Chen F, Chu I K, Che C M, Ho C T, Wang M. Inhibition of mutagenic PhIP formation by epigallocatechin gallate via scavenging of phenylacetaldehyde. Molecular Nutrition \& Food Research, 2009, 53(6): 716-725

42. Zhang X, Chen F, Wang M. Antioxidant and antiglycation activity of selected dietary polyphenols in a cookie model. Journal of Agricultural and Food Chemistry, 2014, 62(7): 1643-1648

43. Zheng Z P, Yan Y, Xia J, Zhang S, Wang M, Chen J, Xu Y. A phenylacetaldehyde-flavonoid adduct, 8-C-(E-phenylethenyl)-norartocarpetin, exhibits intrinsic apoptosis and MAPK pathwaysrelated anticancer potential on HepG2, SMMC-7721 and QGY7703. Food Chemistry, 2016, 197(Pt B): 1085-1092

44. Zhu Q, Zhang S, Wang M, Chen J, Zheng Z P. Inhibitory effects of selected dietary flavonoids on the formation of total heterocyclic amines and 2-amino-1-methyl-6-phenylimidazo[4,5-b]pyridine (PhIP) in roast beef patties and in chemical models. Food \& Function, 2016, 7(2): 1057-1066

45. Cheng K W, Wong C C, Cho C K, Chu I K, Sze K H, Lo C, Chen F, Wang M. Trapping of phenylacetaldehyde as a key mechanism responsible for naringenin's inhibitory activity in mutagenic 2amino-1-methyl-6-phenylimidazo[4,5-b]pyridine formation. Chemical Research in Toxicology, 2008, 21(10): 2026-2034

46. Li H, Zhu F, Chen H, Cheng K W, Zykova T, Oi N, Lubet R A, Bode A M, Wang M, Dong Z. 6-C-(E-phenylethenyl)-naringenin suppresses colorectal cancer growth by inhibiting cyclooxygenase1. Cancer Research, 2014, 74(1): 243-252

47. Nowotny K, Jung T, Höhn A, Weber D, Grune T. Advanced glycation end products and oxidative stress in type 2 diabetes mellitus. Biomolecules, 2015, 5(1): 194-222

48. Ames J M. Applications of the Maillard reaction in the food industry. Food Chemistry, 1998, 62(4): 431-439

49. Bierhaus A, Hofmann M A, Ziegler R, Nawroth P P. AGEs and their interaction with AGE-receptors in vascular disease and diabetes mellitus. I. The AGE concept. Cardiovascular Research, 1998, 37 (3): 586-600

50. Thornalley P J. Dicarbonyl intermediates in the maillard reaction. Annals of the New York Academy of Sciences, 2005, 1043(1): 111117

51. Liu G, Xia Q, Lu Y, Zheng T, Sang S, Lv L. Influence of quercetin and its methylglyoxal adducts on the formation of $\alpha$-dicarbonyl compounds in lysine and glucose model system. Journal of Agricultural and Food Chemistry, 2017, 65(10): 2233-2239

52. Peng X, Ma J, Chen F, Wang M. Naturally occurring inhibitors against the formation of advanced glycation end-products. Food \& Function, 2011, 2(6): 289-301

53. Yu P, Xu X B, Yu S J. Inhibitory effect of sugarcane molasses extract on the formation of $\mathrm{N}(\varepsilon)$-(carboxymethyl)lysine and $\mathrm{N}(\varepsilon)$ (carboxyethyl)lysine. Food Chemistry, 2017, 221: 1145-1150

54. He J, Zeng M, Zheng Z, He Z, Chen J. Simultaneous determination of $\mathrm{N} \varepsilon$-(carboxymethyl) lysine and $\mathrm{N} \varepsilon$-(carboxyethyl) lysine in cereal foods by LC-MS/MS. European Food Research and Technology, 2014, 238(3): 367-374

55. Sasaki K, Chiba S, Yoshizaki F. Effect of natural flavonoids, stilbenes and caffeic acid oligomers on protein glycation. Biomedical Reports, 2014, 2(5): 628-632

56. Chen X Y, Huang I M, Hwang L S, Ho C T, Li S, Lo C Y. Anthocyanins in blackcurrant effectively prevent the formation of advanced glycation end products by trapping methylglyoxal. Journal of Functional Foods, 2014, 8: 259-268

57. Sang S, Shao X, Bai N, Lo C Y, Yang C S, Ho C T. Tea polyphenol (-)-epigallocatechin-3-gallate: a new trapping agent of reactive dicarbonyl species. Chemical Research in Toxicology, 2007, 20(12): $1862-1870$

58. Peng X, Ma J, Cheng K W, Jiang Y, Chen F, Wang M. The effects of grape seed extract fortification on the antioxidant activity and quality attributes of bread. Food Chemistry, 2010, 119(1): 49-53

59. Peng X, Ma J, Chao J, Sun Z, Chang R C C, Tse I, Li E T, Chen F, Wang M. Beneficial effects of cinnamon proanthocyanidins on the formation of specific advanced glycation endproducts and methylglyoxal-induced impairment on glucose consumption. Journal of Agricultural and Food Chemistry, 2010, 58(11): 6692-6696 